\title{
STRANGE BILLIARD TABLES
}

BY

\author{
BENJAMIN HALPERN
}

\begin{abstract}
A billiard table is any compact convex body $T$ in the plane bounded by a continuously differentiable curve $\partial T$. An idealized billiard ball is a point which moves at unit speed in a straight line except when it hits the boundary $\partial T$ where it rebounds making the angle of incidence equal to the angle of reflection. A rather surprising phenomenon can happen on such a table.
\end{abstract}

1. Introduction. The idealized convex billiard table on which an idealized perfectly elastic billiard ball moves with unit speed is a well-known dynamical system [1]-[4]. The table $T$ is any compact convex body in the plane bounded by a continuously differentiable curve $\partial T$ which of course has a unique tangent line at each point. The ball is a point which moves at unit speed in a straight line except when it hits the boundary $\partial T$ where it rebounds making the angle of incidence equal to the angle of reflection. To be precise, a trajectory of the billiard ball is any unit speed rectifiable curve $\alpha(t)$ such that if $\alpha\left(t_{0}\right) \notin \partial T$ then $\alpha(t)$ is part of a straight line for $t$ near $t_{0}$, and if $\alpha\left(t_{0}\right) \in \partial T$ then the left- and right-sided derivatives of $\alpha$ exist at $t_{0}$ and make equal and opposite angles with the tangent to $\partial T$ at $\alpha\left(t_{0}\right)$. It has been observed in [2] that this defines a flow $F_{t}$ on the space of "states of motion". That is, for each initial position $p \in \operatorname{interior} T=\operatorname{int} T$ and direction of motion $v(v \in S=$ set of unit vectors), $F_{t}(p, v)$ gives the position and forward direction of motion of the ball at time $t$.

We will show that $F_{t}$ is not always well defined, and we will construct a billiard table for which it is possible to start a ball in the interior of the table and find it later outside of the table. Our example of a table with $F_{t}$ not well defined is such that the boundary curve has three derivatives and nowhere vanishing curvature. In this example the third derivative exists everywhere, but it is not bounded. We show that if the boundary curve has nowhere vanishing curvature and a bounded third derivative, then $F_{t}$ is well defined. We also show that, for all tables $T, F_{t}(p, v)$ is well defined for almost all $(p, v) \in \operatorname{int} T \times S$.

Received by the editors December 12, 1975 and, in revised form, March 19, 1976.

AMS (MOS) subject classifications (1970). Primary 52A10.

Key words and phrases. Billiard table, plane convex body, dynamical system.

O American Mathematical Society 1977 
The author is indebted to I. Kupka for many stimulating conversations.

2. $F_{t}$ not necessarily well defined. To say that $F_{t_{0}}(p, v)$ is well defined is to assert that there is exactly one trajectory $\alpha(t)$ defined for $t \in\left[0, t_{0}\right]$ such that $\alpha(0)=p$ and $\alpha^{\prime}(0)=v$. It appears that such a unique trajectory always exists for $p \in \operatorname{interior} T$ and that it may be constructed step by step as follows. Travel at unit speed in the direction $v$ starting at $p$ until you hit $\partial T$. Determine the new direction from the equal angles law and proceed at unit speed along a straight line in that direction until you hit $\partial T$ again, and so on. The fallacy in this reasoning is that there is no guarantee that you will use up the time $t_{0}$ in this manner. This resembles Zeno's paradox. We will construct an example which shows that this possibility actually occurs, i.e., the ball can hit the boundary an infinite number of times in a finite amount of time. We will see that at the end of such an infinite sequence of hits the ball is approaching $\partial T$ with zero angle. This leads to at least three ways to continue the trajectory. First, if the table is constructed appropriately the ball could follow a similar infinite hits backwards into the interior of $T$. Second, the ball could follow $\partial T$ around at unit speed. Third, the ball may proceed at unit speed out along the tangent line. Each of these possibilities satisfies our definition of trajectory and hence it is possible for $F_{t}(p, v)$ not to be well defined because the desired trajectory $\alpha$ is not unique. This also explains how a ball starting in the interior of $T$ might end up outside of $T$. The third possibility of continuing tangentially may appear unnatural but the point is that it does satisfy the given definition of trajectory, and this definition looked unobjectionable at first.

3. An example. The construction of the curve $\gamma=\partial T$ such that some trajectory will hit $\gamma$ an infinite number of times in a finite period of time is quite transparent. We first choose the points of contact $p_{n}$ of the special trajectory $\alpha$ and the boundary curve $\gamma$. We choose them on the unit circle with polar angles $\theta_{n}=n^{-1 / 2}$. This determines the polygonal trajectory $\alpha$ and the angle of reflection equals angle of incidence law gives the tangent line to $\gamma$ at $p_{n}$. So we construct a small portion of $\gamma$ through each point $p_{n}$ so as to have the correct tangent at $p_{n}$. We choose these pieces $\gamma_{n}$ to be portions of different unit circles (with appropriate centers) which go through the $p_{n}$. Next we connect up the $\gamma_{n}$ by expressing them via polar equations $\gamma_{n}: r=\bar{\gamma}_{n}(\theta)$ and writing $\gamma: r=\bar{\gamma}(\theta)$ as a smooth average of $\gamma_{n}$ and $\gamma_{n+1}$ for $\theta_{n+1} \leqslant \theta \leqslant \theta_{n}$,

$$
\bar{\gamma}(\theta)=\bar{\gamma}_{n}(\theta)+\alpha_{n}(\theta)\left(\bar{\gamma}_{n+1}(\theta)-\bar{\gamma}_{n}(\theta)\right) .
$$

The averaging functions $\alpha_{n}$ are constructed by

$$
\alpha_{n}(\theta)=\alpha\left(\frac{\theta-\theta_{n+1}}{\theta_{n}-\theta_{n+1}}\right)
$$


where $\alpha:[0,1] \rightarrow[0,1]$ is any fixed (independent of $n$ ) infinitely differentiable function such that $\alpha(t)=1$ for $t \leqslant 1 / 3$ and $\alpha(t)=0$ for $t \geqslant 2 / 3$. Actually we will use the above construction for $\bar{\gamma}$ only for $0<\theta \leqslant \theta_{n_{0}}$ where $n_{0}$ is picked later after making some estimates. Furthermore we redefine $\bar{\gamma}_{n_{0}}$ by setting $\bar{\gamma}_{n_{0}}(\theta)=1$. The other $\bar{\gamma}_{n}$ 's, $n>n_{0}$, are as before. This allows us to complete the definition of $\bar{\gamma}$ by setting $\bar{\gamma}(\theta)=1$ for $\theta \in[-\pi, \pi)-\left(0, \theta_{n_{0}}\right)$.

It remains to be shown that for $n_{0}$ sufficiently large, $\bar{\gamma}$ has three derivatives and nowhere vanishing curvature. To this end we make the following estimates. Taylor expansions immediately give

$$
\delta_{n} \equiv \theta_{n}-\theta_{n-1}=a_{n} n^{-3 / 2}, \quad \omega_{n} \equiv \delta_{n+1}-\delta_{n}=b_{n} n^{-5 / 2},
$$

with $a_{n} \rightarrow-1 / 2$ and $b_{n} \rightarrow 3 / 4$ as $n \rightarrow \infty$. Let $c$ be a bound for $\left|\alpha^{\prime}(t)\right|$ and $\left|\alpha^{\prime \prime}(t)\right|, t \in[0,1]$. Then from (2) and (3)

$$
\begin{aligned}
& \left|\alpha_{n}^{\prime}(\theta)\right| \leqslant c /\left|\delta_{n}\right| \leqslant 3 c n^{3 / 2}, \\
& \left|\alpha_{n}^{\prime \prime}(\theta)\right| \leqslant c / \delta_{n}^{2} \leqslant 5 c n^{3},
\end{aligned}
$$

for $\theta \in\left[\theta_{n+1}, \theta_{n}\right]$ and $n$ sufficiently large.

To obtain good estimates on the $\bar{\gamma}_{n}$ and their derivatives we note that the angle between $S(S=$ the unit circle centered at 0$)$ and $\gamma_{n}$ at $p_{n}$ is $\omega_{n} / 4$. Also we can write

$$
\gamma_{n}(\theta)=g\left(\theta-\theta_{n}, \omega_{n}\right)
$$

where $r=g(\theta, \omega)$ is the polar equation for the unit circle passing through the point $(r, \theta)=(1,0)$ and making an angle of $\omega / 4$ with $S$ at $(1,0)$. It is easy to verify that $g$ is well defined and infinitely differentiable on the compact set $D=\{(\theta, \omega) \mid-\pi / 2 \leqslant \theta \leqslant \pi / 2,-\pi / 4 \leqslant \omega \leqslant \pi / 4\}$. It is also clear that $g(0, \omega)$ $=1=g(\theta, 0)$, for $(\theta, \omega) \in D$. If $e$ is a positive upper bound for the absolute value of both partial derivatives $g_{12}$ and $g_{112}$ over $D$ then the mean value theorem gives

$$
\begin{aligned}
\left|g_{1}(\theta, \omega)\right| & \leqslant e|\omega|, \quad\left|g_{11}(\theta, \omega)\right| \leqslant e|\omega|, \\
|g(\theta, \omega)-1| & \leqslant\left|g_{1}\left(\theta^{*}, \omega\right) \theta\right| \leqslant e|\omega||\theta|,
\end{aligned}
$$

where $\theta^{*}$ is between 0 and $\theta$. Consequently from (5), (6) and (3)

$$
\begin{aligned}
\left|\bar{\gamma}_{n}^{\prime}(\theta)\right| & \leqslant e\left|\omega_{n}\right| \leqslant e\left|b_{n}\right| n^{-5 / 2} \leqslant e n^{-5 / 2}, \\
\left|\bar{\gamma}_{n}^{\prime \prime}(\theta)\right| & \leqslant e\left|\omega_{n}\right| \leqslant e\left|b_{n}\right| n^{-5 / 2} \leqslant e n^{-5 / 2}, \\
\left|\bar{\gamma}_{n}(\theta)-1\right| & \leqslant e\left|\omega_{n}\right|\left|\theta-\theta_{n}\right| \leqslant e\left|\omega_{n}\right|\left|\delta_{n}\right| \leqslant\left|e a_{n} b_{n} n^{-4}\right| \leqslant e n^{-4},
\end{aligned}
$$


for $\theta \in\left[\theta_{n+1}, \theta_{n-1}\right]$ and $n$ sufficiently large.

Now if we pick $n_{0}$ sufficiently large and redefine $\bar{\gamma}_{n_{0}}$ to be given by $\bar{\gamma}_{n_{0}}(\theta)=1$, then from (1), (4) and (7) we easily calculate that

$$
\begin{aligned}
|\bar{\gamma}(\theta)-1| & \leqslant 3 e n^{-4}, \\
\left|\bar{\gamma}^{\prime}(\theta)\right| & \leqslant e n^{-5 / 2}+2 e n^{-5 / 2}+3 c n^{3 / 2} 2 e n^{-4} \leqslant(3 e+6 c e) n^{-5 / 2}, \\
\left|\bar{\gamma}^{\prime \prime}(\theta)\right| & \leqslant e n^{-5 / 2}+2 e n^{-5 / 2}+2 \cdot 3 c n^{3 / 2} 2 e n^{-5 / 2}+5 c n^{3} 2 e n^{-4} \\
& \leqslant 2 \cdot 3 c e n^{-1}, \\
\left|\frac{\bar{\gamma}^{\prime \prime}(\theta)}{\theta}\right| & \leqslant \frac{2 \cdot 3 c e n^{-1}}{(n+1)^{-1 / 2}},
\end{aligned}
$$

for $n \geqslant n_{0}$ and $\theta \in\left[\theta_{n+1}, \theta_{n}\right]$. It follows that $\lim _{\theta \rightarrow 0} \bar{\gamma}(\theta)=1, \lim _{\theta \rightarrow 0} \bar{\gamma}^{\prime}(\theta)$ $=0, \lim _{\theta \rightarrow 0} \bar{\gamma}^{\prime \prime}(\theta)=0$. Hence $\bar{\gamma}^{\prime}(0)$ and $\bar{\gamma}^{\prime \prime}(0)$ exist and $\bar{\gamma}, \bar{\gamma}^{\prime}$ and $\bar{\gamma}^{\prime \prime}$ are continuous. Also $\left(\bar{\gamma}^{\prime \prime}(\theta)-\bar{\gamma}^{\prime \prime}(0)\right) /(\theta-0)=\bar{\gamma}^{\prime \prime}(\theta) / \theta \rightarrow 0$ as $\theta \rightarrow 0$. Hence $\bar{\gamma}^{\prime \prime \prime}(0)$ exists. We also see from (8) that if $n_{0}$ is picked sufficiently large then the curvature of $\gamma$ can be made arbitrarily close to the curvature of $S$ and hence never vanishes. This completes the construction of $\gamma$.

4. Sufficient conditions for $F_{t}$ to be well defined. Consider the discrete analogue $G$ of $F_{t}$ defined as follows. Given a point $p \in \partial T$ and an angle $\theta$, $0<\theta<\pi$, set $G(p, \theta)=\left(p^{\prime}, \theta^{\prime}\right)$ where $p^{\prime}$ is the other intersection of the directed line $L$ which passes through $p$ making an angle $\theta$ with the tangent to $\partial T$ at $p$ ( $\partial T$ is oriented counterclosckwise) and $\theta^{\prime}$ is the positive angle between $L$ and the tangent to $\partial T$ at $p^{\prime}$. That is, if the billiard ball leaves from $p$ making an angle $\theta$ with $\partial T$ then $p^{\prime}$ is the next point of contact with the boundary $\partial T$ and $\theta^{\prime}$ is the next angle of reflection. Since $T$ is convex, $G: \partial T \times(0, \pi)$ $\rightarrow \partial T \times(0, \pi)$ is well defined. Set $G^{n}=G \circ G \circ \cdots \circ G n$ times. Let $P_{1}$ : $\partial T \times(0, \pi) \rightarrow \partial T$ and $P_{2}: \partial T \times(0, \pi) \rightarrow(0, \pi)$ be the natural projections, $P_{1}(p, \theta)=p$ and $P_{2}(p, \theta)=\theta$. Denote the Euclidean norm on the plane $\mathbf{R}^{2}$ by $\|\cdot\|$.

THEOREM 1. If $T$ is any billiard table and $\lim _{n \rightarrow \infty} P_{1} \circ G^{n}(p, \theta)=q$ then

(a) $\lim P_{2} \circ G^{n}(p, \theta)=0$ or $\pi$.

(b) Either $\sum_{n=1}^{\infty} P_{2} \circ G^{n}(p, \theta)<\infty$ or $\sum_{n=1}^{\infty} \pi-P_{2} \circ G^{n}(p, \theta)<\infty$.

(c) $\sum_{n=0}^{\infty}\left\|P_{1} G^{n+1}(p, \theta)-P_{1} G^{n}(p, \theta)\right\|<\infty$.

Proof. Set up a Cartesian coordinate system with the origin at $q$, the positive $x$-axis in the direction of the tangent to $\gamma$ at $q$ and the positive $y$-axis in the direction of the principal normal of $\gamma$ at $q$. We may express the curve $\gamma$ locally about $q$ as $y=g(x)$ for $|x|<\varepsilon$ where $\varepsilon>0$ and $g$ is continuously differentiable and satisfies $g(0)=0, g^{\prime}(0)=0$. Call this portion of $\gamma, \bar{\gamma}$. By 
picking $\varepsilon$ smaller if necessary we may further require that $\left|g^{\prime}(x)\right| \leqslant 10^{-6}$ for $|x|<\varepsilon$. Since $\lim _{n \rightarrow \infty} P_{1} \circ G^{n}(p, \theta)=q$ there is an $n_{0}$ such that $q_{n}=P_{1}$ - $G^{n}(p, \theta) \in \bar{\gamma}$ for $n \geqslant n_{0}$. By replacing $(p, \theta)$ by $G^{n_{0}}(p, \theta)$ we may assume $n_{0}=0$. Set $\theta_{n}=P_{2} \circ G^{n}(p, \theta)$ and $x_{n}$ and $y_{n}$ the $x$ and $y$ coordinates of $q_{n}$. It follows from the mean value theorem that the slope $s$ of the line $\overline{q_{n} q_{n+1}}$ must satisfy $|s| \leqslant 10^{-6}$. We may now show that the sequence $x_{n}$ is monotone. For if the $x_{n}$ 's should change direction $x_{n}<x_{n+1}>x_{n+2}$ or $x_{n}>x_{n+1}<x_{n+2}$ for some $n$, then clearly the tangent to $\gamma$ at $q_{n+1}$ must be nearly vertical. But this is ruled out because $\left|g^{\prime}(x)\right| \leqslant 10^{-6}$ for all $x,|x|<\varepsilon$. Hence the sequence $x_{n}$ is monotone. By reversing the direction of the $x$-axis if necessary we may assume that $x_{n}$ is monotone increasing. Since $\lim _{n \rightarrow \infty} x_{n}=0$ we must have $x_{n} \leqslant 0$. Since the direction of motion monotonically changes by $2 \theta_{i}$ at each point of contact, $\sum_{i=1}^{\infty} 2 \theta_{i}$ is equal to the angle between the initial direction of motion and the tangent at $q$. Conclusions (a), (b), and $\sum_{n=1}^{\infty}\left\|q_{n+1}-q_{n}\right\| \leqslant$ arclength of $\gamma$ from $q_{1}$ to $q$, and (c) now follows easily.

Corollary 2. Given $(p, \theta) \in \partial T \times(0, \pi)$ and $p_{0}$ strictly between $p$ and $q=P_{1} G(p, \theta)$ on the straight line segment from $p$ to $q$, set $v=(q-p) /\|q-p\|$. The following are equivalent.

(a) $F_{t}\left(p_{0}, v\right)$ is well defined for all $t \geqslant 0$.

(b) $\sum_{n=0}^{\infty}\left\|P_{1} G^{n+1}(p, \theta)-P_{1} G^{n}(p, \theta)\right\|=\infty$.

(c) $P_{1} G^{n}(p, \theta)$ diverges.

Proof. First we show not (c) $\Rightarrow$ not (a). Assume $P_{1} G^{n}(p, \theta)$ converges. By Theorem 1,

$$
\sum_{n=0}^{\infty}\left\|P_{1} G^{n+1}(p, \theta)-P_{1} G^{n}(p, \theta)\right\|<\infty
$$

and $P_{2} G^{n}(p, \theta) \rightarrow 0$ or $\pi$. Hence starting at $(p, \theta)$ the ball is approaching a point $q \in \partial T$ tangentially at a finite time $t_{0}$. Since we may continue the trajectory in at least two ways, around $\partial T$ or out along the tangent line, $F_{t}\left(p_{0}, v\right)$ is not well defined for $t>t_{0}$.

Second we note that not (b) $\Rightarrow$ not (c) follows immediately from Cauchy's convergence criteria.

Third we show that (b) $\Rightarrow(a)$. Assuming (b) the naive construction and uniqueness proof discussed in $\$ 2$ goes through and $F_{t}\left(p_{0}, v\right)$ is well defined for all $t \geqslant 0$.

The equivalence of (a), (b) and (c) follows. Q.E.D.

We will need some notation for Theorem 3 and its proof. The curvature of $\gamma=\partial T$ at the value of arclength $s$ is denoted by $K(s)$. The principal normal to $\gamma$ at $s$ is denoted by $n(s)$. For any function of the arclength $r(s)$ we write $\dot{r} \equiv d r / d s$. We consider $\mathbf{R}^{2}$ imbedded as $\mathbf{R}^{2} \times 0$ in $\mathbf{R}^{3}$ and then by identifying 
the third axis with $\mathbf{R}$ we can consider the vector cross product, denoted by $[\cdot, \cdot]$, as a function from $\mathbf{R}^{2} \times \mathbf{R}^{2}$ into $\mathbf{R}$. We take the curve $\gamma$ to be a periodic function $\gamma: \mathbf{R} \rightarrow \mathbf{R}^{2}$ with period $=$ length $\gamma$. We will use $o(t-s)^{2}$ to signify any function $f(s, t)$ such that there exists a constant $L$ such that $|f(s, t)|$ $\leqslant L(t-s)^{2}$ for all $s, t \in \mathbf{R}$.

THEOREM 3. If $\partial T$ has a bounded third derivative and nowhere vanishing curvature, then $F_{t}(p, v)$ is well defined for all $(p, v) \in \operatorname{int} T \times S$.

Proof. Assume the hypothesis and the contrary to the conclusion. It follows that $\dot{K}(s)$ exists and is bounded, where $K(s)$ is the curvature at $s$. By Corollary 2 and Theorem 1 there is a $p \in \partial T$ and $\theta, 0<\theta<\pi$, such that

$$
P_{1} G^{n}(p, \theta) \text { converges, }
$$

$$
\sum_{n} P_{2} G^{n}(p, \theta)<\infty
$$

(We reverse the parametrization of $\gamma=\partial T$ if necessary.) Set $\theta_{n}=P_{2} G^{n}(p, \theta)$. Using (9) we will show that there exist an $L$ and an $n^{\prime}$ such that, for each $n \geqslant n^{\prime}$,

$$
\theta_{n+1}=\theta_{n}+d_{n} \theta_{n}^{2}
$$

for some $d_{n}$ such that $\left|d_{n}\right| \leqslant L$. Assume this for the time being.

We will show how this leads to a contradiction of (10).

A theorem of Weierstrass [5, p. 133] states that if the terms $a_{n}$ of a given series $\sum a_{n}$ of complex terms possess for $n \geqslant m$ a representation of the form

$$
a_{n+1} / a_{n}=1-\alpha / n+c_{n}
$$

with $\sum\left|c_{n}\right|<\infty$, then $\sum a_{n}$ is convergent if and only if the real part of $\alpha>1$. We apply this result with $a_{n}=\theta_{n}, m=n^{\prime}, c_{n}=d_{n} \theta_{n}$, and $\alpha=0$. From (10) we have $\sum \theta_{n}<\infty$. Since $\left|d_{n}\right| \leqslant L$ for $n \geqslant n^{\prime}$ we have $\sum_{n=n^{\prime}}^{\infty}\left|c_{n}\right|$ $=\sum_{n=n^{\prime}}^{\infty}\left|d_{n} \theta_{n}\right| \leqslant \sum_{n=n^{\prime}}^{\infty} L \theta_{n}<\infty$. From (11) we have

$$
\theta_{n+1} / \theta_{n}=1-0 / n+c_{n} \text {. }
$$

Hence Weierstrass' theorem implies that $\Sigma \theta_{n}$ diverges. This contradicts (10).

It remains to establish (11). Consider two values of the arclength, $s<t$. Let $\alpha$ and $\beta$ be, respectively, the small positive angles made by $\dot{\gamma}(s)$ and $\dot{\gamma}(t)$ with $\gamma(t)-\gamma(s)$. Expanding $\gamma$ in a Taylor series about $s$ we obtain 


$$
\begin{aligned}
\sin \alpha & =\frac{[\dot{\gamma}(s), \gamma(t)-\gamma(s)]}{\|\gamma(t)-\gamma(s)\|} \\
& =\frac{\left[\dot{\gamma}(s), \dot{\gamma}(s)(t-s)+\frac{1}{2} \ddot{\gamma}(s)(t-s)^{2}+\frac{1}{6} \ddot{r}(\xi)(t-s)^{3}\right]}{\left\|\dot{\gamma}(s)(t-s)+\frac{1}{2} \ddot{\gamma}(\eta)(t-s)^{2}\right\|} \\
& =\frac{1}{2} K(s)(t-s)+o(t-s)^{2}
\end{aligned}
$$

where $s \leqslant \xi, \eta \leqslant t$ and we have used $\ddot{\gamma}(s)=K(s) n(s)$ and $\ddot{\gamma}(\xi)=\dot{K}(\xi) n(\xi)$ $-(K(\xi))^{2} \dot{\gamma}(\xi)$. Similarly

$$
\sin \beta=\frac{1}{2} K(t)(t-s)+o(t-s)^{2} .
$$

Hence by expanding arcsin in a Taylor series we have

$$
\alpha=\frac{1}{2} K(s)(t-s)+o(t-s)^{2}, \quad \beta=\frac{1}{2} K(t)(t-s)+o(t-s)^{2} .
$$

Thus

$$
\begin{aligned}
\beta-\alpha & =\frac{1}{2}(K(t)-K(s))(t-s)+o(t-s)^{2} \\
& =\frac{1}{2} \dot{K}(\delta)(t-s)^{2}+o(t-s)^{2}
\end{aligned}
$$

where $s \leqslant \delta \leqslant t$. Therefore

$$
|\beta-\alpha| \leqslant L_{1}(t-s)^{2}
$$

for some constants $L_{1}$ independent of $s$ and $t$.

Since $K$ is continuous periodic and always positive there must be by compactness a $\Delta>0$ such that $K(r) \geqslant \Delta$ for all $r \in R$. In light of this it follows from (12) and (13) that there exist an $\varepsilon>0$ and an $L$ such that

$$
|\beta-\alpha| \leqslant L \alpha^{2} \text { for all } s, t \text { such that }|t-s| \leqslant \varepsilon .
$$

Equation (11) now follows from (9) and (14). Q.E.D.

REMARK. The proof of Theorem 3 shows that $\lim _{n \rightarrow \infty} P_{1} G^{n}(p, \theta)=q$ is impossible if $K>0$ at $q$ and $\gamma^{\prime \prime \prime}$ is bounded in a neighborhood of $q$.

We will use $m$ to represent each of the following measures: Lebesgue measure on int $T \subset \mathbf{R}^{2}$ and on $(0, \pi) \subset \mathbf{R}^{1}$, arclength measure on the unit circle $S$ and on $\partial T$, and the product measures $m \times m$ on int $T \times S$ and on $\partial T \times(0, \pi)$.

THEOREM 4. On any billiard table $T$, for almost all initial conditions $(p, v)$ $\in$ int $T \times S, F_{t}(p, v)$ is well defined for all $t \geqslant 0$.

Proof. Assume $m(Q)>0$ where $Q=\left\{(p, v) \in\right.$ int $T \times S \mid F_{t}(p, v)$ is not 
well defined for some $t \geqslant 0$ \}. Birkhoff shows in [3] that the measure $\mu$ on $\partial T \times(0, \pi)$ given by

$$
\mu(A)=\int_{A} \sin \theta d m(p) d m(\theta)
$$

( $A$ an $m$-measurable subset of $\partial T \times(0, \pi)$ ) is preserved by $G$. The proof of this fact involves calculating the Jacobian of $G$ which is found to be $\sin \theta / \sin \theta_{1}$ where $\theta_{1}=P_{2} G(p, \theta)$. From this it also can be seen that the rank of $H$ : int $T \times S \rightarrow \partial T \times(0, \pi)$ is always 2 where $H(p, v)=(q, \theta)$ as shown in Figure 1.

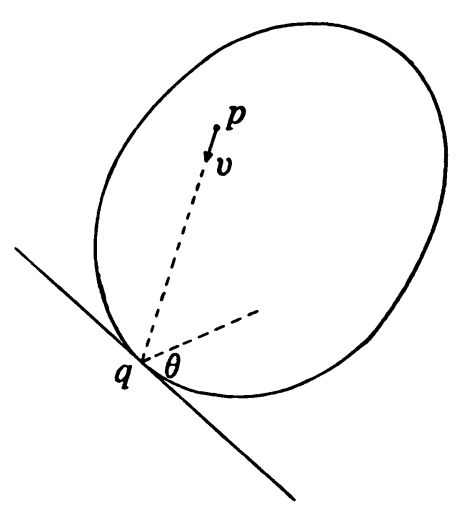

FIGURE 1

Hence $H$ is locally like a projection of $\mathbf{R}^{3}$ onto $\mathbf{R}^{2}$. It follows that $m(R)>0$ where $R=H(Q)=\left\{(q, \theta) \in \partial T \times(0, \pi) \mid P_{1} G^{n}(q, \theta)\right.$ converges as $\left.n \rightarrow \infty\right\}$. The second equality comes from Corollary 2 . It follows from (15) that $\mu(R)>0$. Let

$$
U_{\varepsilon}=\{(q, \theta) \in \partial T \times(0, \pi) \mid \theta<\varepsilon \text { or } \pi-\theta<\varepsilon\} .
$$

Clearly $\mu\left(U_{\varepsilon}\right) \rightarrow 0$ as $\varepsilon \rightarrow 0$. Pick $\varepsilon$ so that $\mu\left(U_{\varepsilon}\right)<\frac{1}{2} \mu(R)$. By Corollary 2 and Theorem 1, for each $(p, \theta) \in R, P_{2} G^{n}(p, \theta) \rightarrow 0$ or $\pi$. Hence, for every $(p, \theta) \in R$ there is an $n$ such that $G^{n^{\prime}}(p, \theta) \in U_{\varepsilon}$ for all $n^{\prime} \geqslant n$. Therefore

$$
R \subset \bigcup_{n=1}^{\infty} \bigcap_{n^{\prime} \geqslant n} G^{-n^{\prime}}\left(U_{\varepsilon}\right)
$$

and the union is an increasing one. Hence we can find an $n$ such that

$$
\mu\left(\bigcap_{n^{\prime}>n} G^{-n^{\prime}}\left(U_{\varepsilon}\right)\right)>\frac{1}{2} \mu(R) .
$$

Thus, $\mu\left(G^{-n}\left(U_{\varepsilon}\right)\right)>\frac{1}{2} \mu(R)$. But $G$ preserves $\mu$, so $\mu\left(G^{-n}\left(U_{\varepsilon}\right)\right)=\mu\left(U_{\varepsilon}\right)$ 
$>\frac{1}{2} \mu(R)$, a contradiction. Therefore $m Q=0$. Q.E.D.

We leave the following trick shot to the reader. Given a table $T$ with $F_{t}$ not well defined and a point $q$ outside the table, start the ball in the interior of $T$ and hit the point $q$.

\section{REFERENCES}

1. George D. Birkhoff, Some unsolved problems of theoretical dynamics, Science 94 (1941), 598-600. MR 3, 279.

2. —- What is the ergodic theorem? Amer. Math. Monthly 49 (1942), 222-226. MR 4, 15.

3. - On the periodic motions of dynamical systems, Acta Math. 50 (1927), 354-379.

4. H. T. Croft and H. P. F. Swinnerton-Dyer, On the Steinhaus billiard table problem, Proc. Cambridge Philos. Soc. 59 (1963), 37-41. MR 26 \#2925.

5. Konrad Knopp, Infinite sequences and series, Dover, New York, 1956. MR 18, 30.

Department of Mathematics, Indiana University, Bloomington, Indiana 47401 